\title{
Estudio Descriptivo de las Cirugías y Hospitalizaciones en Cirugía Maxilofacial Durante la Pandemia en Chile. Experiencia del Hospital Barros Luco Trudeau
}

\author{
Descriptive Study of the Surgeries and Hospitalizations in Maxillofacial Surgery \\ During the Pandemic in Chile. Barros Luco Trudeau Hospital Experience
}

\author{
Araceli Raposo1,2; Cristián Nuñez ${ }^{1,2}$; Guillermo Quezada ${ }^{1,2}$; Leonardo Quezada1 \& Alexis Carraso1
}

RAPOSO, A; NUÑEZ, C.; QUEZADA, G.; QUEZADA, L. \& CARRASO, A. Estudio descriptivo de las cirugías y hospitalizaciones en Cirugía Maxilofacial durante la pandemia en Chile. Experiencia del Hospital Barros Luco Trudeau. Int. J. Odontostomat., 15(1):71-76, 2021.

RESUMEN: Desde la aparición del SARS-CoV-2 se ha establecido, en la mayoría de los países afectados por esta pandemia, la suspensión de toda cirugía electiva, centrando la atención solo a urgencias. Son varios los estudios que mencionan las recomendaciones necesarias, sin embargo, hasta el minuto existe poca evidencia de la cantidad y tipo de cirugías realizadas por los servicios de Cirugía Maxilofacial. El objetivo del presente trabajo es dar a conocer la epidemiología de los pacientes tratados por el equipo de cirugía maxilofacial en el Hospital Barros Luco Trudeau de Santiago, Chile. Se realizó un estudio de corte transversal entre Marzo y Agosto del 2020, de todos los paciente atendidos por la unidad de Cirugía Maxilofacial del Hospital. Se analizó datos epidemiológicos como también el tipo de patología, la etiología, días de estadía hospitalaria y días de espera para cirugía. La muestra estuvo constituida por 41 pacientes, $20 \%$ mujeres y $80 \%$ hombres. El rango etario fue de 17 a 92 años, con un promedio de 40,39 años (DE 17,67). En al diagnóstico, el 61 \% correspondió a patología traumática y el $39 \%$ a patología infecciosa. El trauma más frecuente fue la fractura mandibular parasinfisiaria (23\%) y la infección más frecuente, la infección secundaria a quistes y tumores (25 \%). A pesar del periodo de pandemia con las restricciones asociadas a la cuarentena, la muestra se comporta similar a estudios previamente reportados tanto en la distribución por sexo y edad, así como en el tipo el trauma. infección.

PALABRAS CLAVE: COVID-19, SARS- CoV-2, cirugía oral y maxilofacial, epidemiología, trauma maxilofacial,

\section{INTRODUCCIÓN}

En diciembre de 2019, se informó de un brote de enfermedad respiratoria causado por un nuevo coronavirus en Wuhan, China, denominado en ese entonces 2019-nCoV (Yang et al., 2020). En poco tiempo se ha convertido en una pandemia mundial, estando presente actualmente en alrededor de 100 países, cobrando más de 1 millón de vidas (World Health Organization, 2020). Hoy el virus es denominado SARSCoV-2 y la enfermedad que produce COVID-19.

Esta situación ha afectado de forma considerable a los trabajadores de salud al estar inmersos en hospitales o clínicas con pacientes COVID-19+. De hecho, se ha reportado que en etapas tempranas de la pandemia los trabajadores de esta área ocupaban el 29 $\%$ de los contagios (Zimmermann \& Nkenke, 2020).

Los cirujanos maxilofaciales como profesionales del área de la salud son particularmente vulnerables a la infección de COVID-19 debido a su exposición a la cavidad oral y nasal, y fluidos corporales de los pacientes, en la práctica clínica habitual (Yang et al.).

Por esta razón se decretó, en la mayoría de los países afectados por esta pandemia, la suspensión de toda cirugía electiva, centrando la atención única-

\footnotetext{
1 Unidad de Cirugía y Traumatología Maxilofacial, Servicio de Cirugía, Hospital Barros Luco Trudeau, Santiago, Chile.

2 Departamento de Cirugía y Traumatología Bucal y Maxilofacial, Facultad de Odontología, Universidad de Chile, Santiago, Chile.
}

Received: 2020-11-03 Accepted: 2020-11-28 
mente a los pacientes de urgencias como el trauma maxilofacial, infecciones y patología dolorosa.

Un estudio realizado por Maffia et al. (2020), entre marzo y abril de 2020, midió a través de una encuesta aplicada a nivel mundial, la situación de los cirujanos maxilofaciales. Este estudio mostró que solo un $3,8 \%$ de los departamentos seguían trabajando normalmente, un 78,2 \% seguían trabajando pero con restricciones y un $17,9 \%$ no estaba atendiendo.

De todas las cirugías que permanecían realizándose por cada centro la más frecuente fue el trauma maxilofacial (66,7 \%) (Maffia et al.). Van der Tas el al. (2020), reportaron que solo el $13,3 \%$ de los cirujanos maxilofaciales se encontraba realizando cirugías electivas, cifra que baja a $6,2 \%$ en Chile.

Respecto a las medidas de protección del personal de salud, con el tiempo se fue implementando protocolos claros de uso de elementos de protección personal (EPP), sin embargo, en los primeros meses de pandemia se produjo una rápida escasez y dificultad para conseguirlos.

Es así como el estudio realizado por Van der Tas et al., demostró que el impacto de COVID-19 en los cirujanos maxilofaciales era global, donde el $57,3 \%$ reportó que sus hospitales no proporcionaban los EPP adecuados, lo que a nivel sudamericado se elevó al 66,7 \%.

Hoy en día el panorama es más claro, los procedimientos quirúrgicos que involucran las regiones de la mucosa nasal-oral tienen un mayor riesgo de infección del personal médico debido a la aerolización del SARSCoV-2 y considerando el porcentaje de pacientes asintómaticos el riesgo es permanente. Debido a esto la recomendación es usar EPP apropiados durante todos los procedimientos quirúrgicos y las visitas ambulatorias, que puede incluir mascarillas FFP2 / N95 y protector facial completo (Grant et al., 2020).

Se han publicado una serie de recomendaciones respecto a la atención de pacientes en cirugía maxilofacial durante este periodo de pandemia (Bali \& Chaudhry, 2020; Piombino et al., 2020; GonzálezQuintanilla \& Santos-López 2020; Melián-Rivas et al., 2020), existiendo claridad en la necesidad de categorización de los pacientes para atender las urgencias reales y así minimizar el riesgo de contagio de pacientes hacia el personal de salud y viceversa, así como también contribuir al confinamiento necesario decretado por las autoridades locales y no fomentar el tránsito de las personas desde y hacia los recintos de salud.

Sin embargo, hasta el minuto existe poca evidencia de la cantidad y tipo de cirugías que se están realizando efectivamente en los distintos servicios de Cirugía Maxilofacial. El objetivo del presente trabajo es dar a conocer la epidemiología de los pacientes tratados por el equipo de cirugía maxilofacial en el Hospital Barros Luco Trudeau en el período Marzo - Agosto de 2020.

\section{MATERIAL Y MÉTODO}

Se realizó un estudio descriptivo, de tipo corte transversal durante el periodo comprendido entre el 16 marzo de 2020 (cuando Chile entró a fase 3 de la pandemia por SARS-CoV-2) al 31 de Agosto de 2020 (donde comienza el desconfinamiento paulatino de la Región Metropolitana).

La población objetivo fueron todos los pacientes ingresados tanto para cirugía como para hospitalización a cargo del equipo de cirugía maxilofacial del Hospital Barros Luco Trudeau, Chile.

Se excluyeron los pacientes que decidieron alta voluntaria (no médica) durante la hospitalización, ya sea previo o posterior a la cirugía y/o tratamiento hospitalario, además de los pacientes que tuvieron hospitalizaciones prolongadas por causas ajenas a la patología maxilofacial.

Los datos fueron extraídos de planilla estadística de uso interno del equipo de cirugía maxilofacial, previa anonimización de los datos de identificación de los pacientes como nombre, rut y número de ficha, los que fueron reemplazados por códigos alfa numéricos. Esta información fue levantada a planilla excel considerando las variables: sexo, edad, diagnóstico de ingreso, etiología, días de hospitalización y días de espera para cirugía. Para calcular en la planilla original los días de espera para cirugía se tomó en cuenta el ingreso realizado por cualquiera de los profesionales del equipo hasta el día de la cirugía.

\section{RESULTADOS}

La muestra estuvo constituida por 41 pacientes, $20 \%$ mujeres $(n=8)$ y $80 \%$ hombres $(n=33)$. El rango etario fue de 17 a 92 años, con un promedio de 40,39 años (DE 17,67). 
En cuanto a la clasificación diagnóstica el $61 \%(n=25)$ correspondió a patología traumática y el $39 \%(n=16)$ a patología infecciosa.

La patología infecciosa más frecuente fue la infección de quistes y/o tumores con un $25 \%(n=4)$, seguido del flegmón perimandibular con un $18,75 \%(n=3)$ (Fig. 1).

Las causas más frecuentes de infecciones fueron complicaciones pulpares $31,25 \%(n=5)$ y los tumores o quistes $25 \%$ $(n=4)$, seguidos de las inclusiones dentarias $12,5 \%(n=3)$. La infección de sitio quirúrgico, fistula buco-sinusal y osteomielitis correspondieron al $6,25 \%(n=1)$ cada una.

Los 25 pacientes ingresados por patología traumática registraron un total de 43 fracturas.

La más frecuente fue la fractura mandibular parasinfisiaria con un $23,26 \%(n=10)$, seguido de fractura de ángulo mandibular $20,93 \%(n=9)$, fractura de cóndilo mandibular $13,95 \%(n=6)$, fractura orbito-cigomática $13,95 \%(n=6)$ y fractura maxilar $13,95 \%$ $(n=6)$ (Fig. 2).

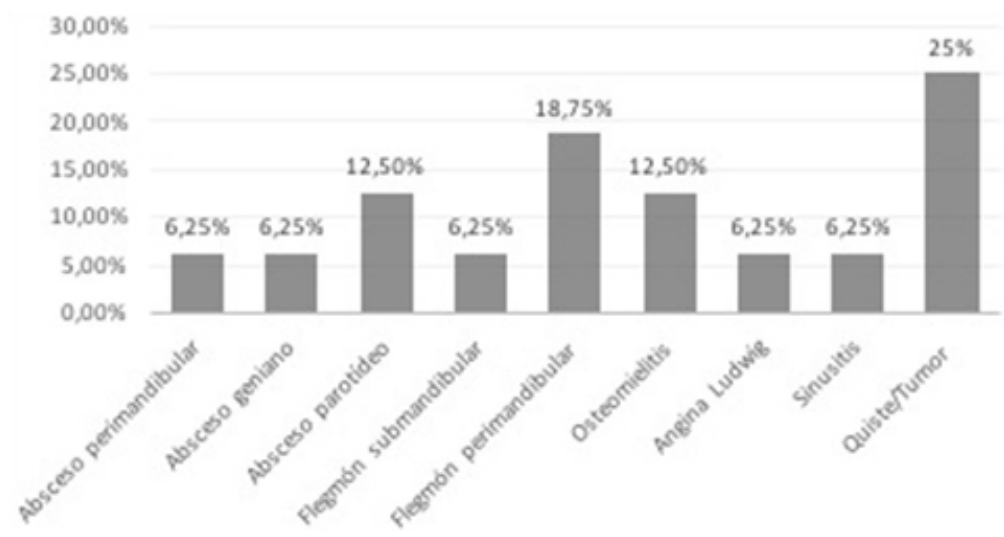

Fig. 1. Distribución por tipo de infección.

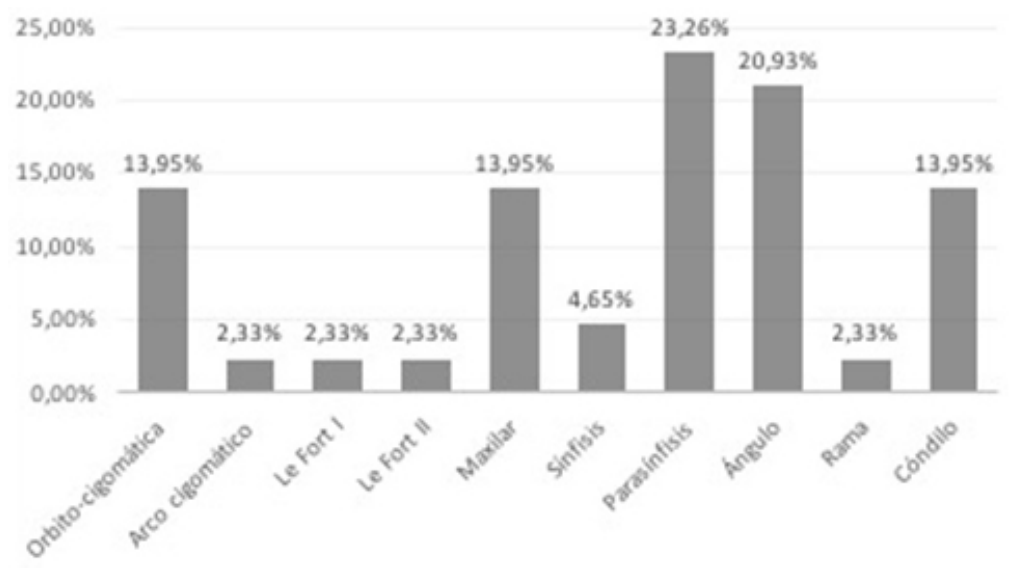

Fig. 2. Distribución por tipo de fractura.
La combinación de fracturas más frecuente fue la fractura orbito-cigomática con fractura maxilar con un $20 \%(n=5)$ seguido de fractura paramediana con ángulo mandibular y paramediana con cóndilo mandibular con un $12 \%(n=3)$ cada una.

En cuanto a las etiologías de las fracturas lo más frecuente fue la agresión física con un $64 \%(n=16)$, seguido de accidentes de tránsito $20 \%(n=5)$ y con menor porcentaje las caídas con un $8,7 \%$ $(n=2)$. De la agresión física, el $50 \%$ correspondió a peleas $(n=8), 25 \%(n=4)$ a violencia intrafamiliar y $25 \%(n=4)$ a asalto.

Se registraron complicaciones en el $14,64 \%$ de los casos, las cuales correspondieron a infección de sitio quirúrgico, complicación neurológica de tipo motora y dehiscencia de herida con un 4,88\% $(n=2)$ cada una.

El rango de días de espera para cirugía fue de 0 a 17 con un promedio de 5,93 días $(\mathrm{DE}=4,79)$. Los días de hospitalización variaron de 1 a 22 días con un promedio de 3,78 días $(\mathrm{DE}=3,66)$.

\section{DISCUSIÓN}

La práctica maxilofacial en nuestro servicio se ha seguido realizando por la demanda de atención de urgencia de los pacientes. En este sentido son muchos los artículos que proponen en el contexto actual, la realización de teleconsultas o triage telefónico previo a la atención de los pacientes (Bali \& Chaudhry), sin embargo, nuestro equipo al pertenecer a un Hospital público de alta complejidad debe atender a toda la población que consulta. Al ingreso de la hospitalización se realizó test PCR y cuestionario a la mayoría de los pacientes decidiendo ingreso a sala COVID +, contacto COVID o sala limpia. De igual forma los pacientes que requerían cirugía se atendieron en pabellón COVID + o en pabellón libre de COVID. Ambos pabellones ubicados en áreas dis- 
RAPOSO, A; NUÑEZ, C.; QUEZADA, G.; QUEZADA, L. \& CARRASO, A. Estudio descriptivo de las cirugías y hospitalizaciones en Cirugía Maxilofacial durante la pandemia en Chile. Experiencia del Hospital Barros Luco Trudeau. Int. J. Odontostomat., 15(1):71-76, 2021

tintas del mismo hospital. Si bien en ambos se utilizaban las mismas EPP, en el pabellón COVID + el protocolo fue mucho más estricto.

Respecto al tipo de pacientes tratados, la distribución hombre mujer se mantiene en relación a reportes previos de la literatura internacional reciente $(\mathrm{Be}-$ llo et al., 2017; Marano et al., 2020) y reportes previos de Chile (Pacheco et al., 2012; Raposo et al., 2013; Pérez et al., 2015), con un predominio notable de hombres. El promedio encontrado en otras series es variable dependiendo si el servicio es solo adultos o adultos y pediatría, fluctuando desde los 16 años (Bello et al.) a los 40 años (Marano et al.), siendo este último promedio similar al encontrado en nuestro estudio.

En cuanto a la distribución por patología traumática e infecciosa, hay poca literatura que abarque ambas áreas. La mayoría de los estudios se centran en el trauma maxilofacial, sin embargo, un estudio realizado en Chile por Pérez et al., reportó un 56 $\%$ de trauma vs un $44 \%$ de patología no traumática que incluía infecciones en su mayoría, seguido de tumores y dismorfosis dentofaciales. Esto es concordante con nuestro estudio donde el trauma ocupó el $61 \%$ de los casos y el 39 \% correspondió a patología infecciosa. En nuestra serie no se presentan dismorfosis dentofaciales ya que al ser patología electiva se encontraba suspendida durante el periodo en estudio. Por otro lado, los tumores/quistes sólo se ingresaron o intervinieron cuando se asociaron a infección.

Según el sitio anatómico de las infecciones, el estudio de Yuvaraj (2016) realizado en India, reportó como más frecuentemente comprometido el espacio pterigomandibular (48\%) seguido del submandibular (21\%) y la etiología más común los terceros molares incluidos (40\%). En el mismo país, Keswani \& Venkaleshwar (2019), reportan el espacio submandibular como el más afectado $(40,63 \%)$ y la etiología más común la patología pulpar $(69,52 \%)$. Este último dato concuerda con nuestro estudio donde la caries (patología pulpar) fue la etiología más encontrada, sin embargo, el porcentaje se reduce a la mitad. A diferencia de estos estudios encontramos un mayor número de infecciones asociadas a patología no odontogénica, como infecciones secundarias a sialolitiasis o infecciones en quistes y tumores, lo que llama la atención, ya que, al haber cesado gran parte de la atención de odontológica, era esperable encontrar infecciones relacionadas al no tratamiento oportuno de las patologías como caries o dientes incluidos.
Al comparar los tipos de fracturas, Lee et al. (2017) en Malasia reportaron como más frecuente la fractura mandibular, específicamente la fractura parasínfisiaria, al igual que nuestro estudio. Marano et al., en Brasil reportó mayor número de fracturas del complejo cigomático y de las fracturas mandibulares reportó como más frecuente la de cóndilo mandibular. A nivel nacional Pérez et al. el 2015 encontraron mayor prevalencia de fractura mandibular $(49,8 \%)$ al igual que nuestro estudio, sin embargo no especifican el subtipo. Raposo et al., en el 2013, encontraron un 27,9 $\%$ de fracturas de ángulo, siendo la más prevalente, seguida de fractura parasinfisiaria con un $20,9 \%$, valores muy similares a los encontrados en nuestra muestra $(20,93 \%$ y $23,26 \%$ respectivamente).

La etiología más frecuente fue la agresión física (64\%), que en su mayoría se producía por peleas. La violencia intrafamiliar es mayor a lo visto por el equipo en condiciones de normalidad, lo que pudiera estar relacionado al confinamiento decretado por las autoridades en el contexto de pandemia. Lamentablemente el tamaño muestral es muy pequeño para sacar conclusiones significativas.

Al comparar estas etiologías con otras series los resultados son distintos, Lee et al., reportan como más frecuente los accidentes automovilísticos con un $65,7 \%$, Marano et al., reportó un 33,7 \% de accidentes de tránsito y en tercer lugar la agresión física con un $16,8 \%$. De los accidentes de tránsito la mayoría se producía por accidentes en motocicleta $(49,2 \%)$, lo que concuerda con nuestro estudio donde todos los pacientes cuya etiología fue accidente de tránsito fue por uso de motocicletas.

Al comparar las etiologías con estudios nacionales, la violencia o agresión ocupan el primer lugar. Raposo et al. reportaron violencia en el $50,3 \%$ de los casos, seguido de accidentes automovilísticos con un $21 \%$. El estudio de Pacheco et al. encontró un 49,1\% de agresiones.

De las complicaciones Marano et al., reportaron un numero inferior, $7,4 \%$, sin embargo, destacan que en la literatura este rango fluctúa entre el 11 al $12,8 \%$, valor que se acerca más a lo encontrado en nuestra serie $(14,64 \%)$.

En relación con la ocupación de camas durante este periodo, se intentó su uso por el menor tiempo posible, siendo las estadías más prolongadas las relacionadas a manejo antibiótico de infecciones y las 
relacionadas a trauma de mayor severidad, como se mencionó anteriormente el promedio fue de 3,7 días de estadía.

En relación con la espera de cirugía, el promedio de 5,9 días es menor a lo usual en nuestro hospital, lo que se debió a la mayor disponibilidad de pabellones por suspensión de la cirugía electiva.

Cabe mencionar que, durante este periodo, se suspendió la pasantía de alumnos y becados en formación por la mayoría de los servicios del hospital incluyendo el nuestro. Las recomendaciones internacionales sugieren que los procedimientos deben ser realizados por cirujanos experimentados, con un número mínimo de asistentes posibles (Grant et al.). De esta manera se acortan los tiempos quirúrgicos, hay menor tasa de complicaciones post-operatorias y por ende estadía hopitalaria más corta.

\section{CONCLUSIONES}

Las patologías usulmente vistas en los servicios de cirugía maxilofacial se mantuvieron constantes durante el periodo de pandemia. Llama la atención que a pesar de la cuarentena obligatoria, el trauma se mantiene como la patología más frecuente, asociada en su mayoria a accidentes automovilísicos y a agresión física. Por otro lado la patología infecciosa de tipo odontogénica no sufrió un aumento importante, a pesar de haber menor disponibilidad de atención odontológica electiva.

En cuanto a la epidemiología propiamente tal, la muestra se comporta muy similar a lo encontrado en otros estudios tanto nacionales como internacionales en cuanto a distribución por sexo y edad, así como con el tipo de trauma.

RAPOSO, A; NUÑEZ, C.; QUEZADA, G.; QUEZADA, L. \& CARRASO, A. Descriptive study of the surgeries and hospitalizations in Maxillofacial Surgery during the pandemic in Chile. Barros Luco Trudeau Hospital Experience. Int. J. Odontostomat., 15(1):71-76, 2021.

ABSTRACT: Since the appearance of SARS-CoV-2, the suspension of all elective surgery has been established in most of the countries affected by this pandemic, focusing only on emergency. There are several studies that mention the necessary recommendations, however, until now there is little evidence of the amount and type of surgeries performed by the different Maxillofacial Surgery services. The objective of this study is to present the epidemiology of the patients treated by the maxillofacial surgery team at the Barros Luco Trudeau Hospital of Santiago, Chile. Material and method: A cross-sectional study was carried out between April and August 2020, of all the patients treated by the Maxillofacial Surgery unit of the Hospital. Epidemiological data were analyzed as well as the type of pathology, the etiology, days of hospital stay and days of waiting for surgery. Results: The sample consisted of 41 patients, $20 \%$ women and $80 \%$ men. The age range was from 17 to 92 years, with an average of 40.39 years (SD 17.67). At diagnosis, $61 \%$ corresponded to traumatic pathology and $39 \%$ to infectious pathology. The most frequent trauma was the parasymphyseal mandibular fracture $(23 \%)$ and the most frequent infection, infection secondary to cysts and tumors $(25 \%)$. Despite the pandemic period with the restrictions associated with quarantine, the sample behaves similar to previously reported studies, both in the distribution by sex and age, as well as in the type of trauma.

KEY WORDS: COVID-19, SARS- CoV-2, oral and maxillofacial surgery, epidemiology, maxillofacial injuries, Infection.

\section{REFERENCIAS BIBLIOGRÁFICAS}

Bali, R. K. \& Chaudhry, K. Maxillofacial surgery and COVID-19, The Pandemic !! J. Maxillofac. Oral Surg., 19(2):159-61, 2020.

Bello, S. A.; Osodin, T.; Oketade, I.; Ibikari, A. B.; Ighile, N.; Enebong, D. J. \& Ekpa, P. Pattern of maxillofacial surgical conditions in North Central Nigeria: A 5-year experience of an indigenous surgical mission. Niger. J. Clin. Pract., 20(10):1283-8, 2017.

González-Quintanilla, D. \& Santos-López, M. Preventive measures and considerations for oral surgery practice during COVID-19. Int J. Odontostomat., 14(3):338-41, 2020.

Grant, M.; Buchbinder, D.; Dodson, T. B.; Fusetti, S.; Leung, M. Y. Y.; Sanchez Aniceto, G.; Schramm, A.; Strong, B. \& Willis, E. AO CMF International Task Force Recommendations on Best Practices for Maxillofacial Procedures During COVID-19 Pandemic, 2020. Craniomaxillofac. Trauma Reconstr., 13(3):151-6, 2020.

Keswani, E. S. \& Venkateshwar, G. Odontogenic maxillofacial space infections: a 5-year retrospective review in Navi Mumbai. J. Maxillofac. Oral Surg., 18(3):345-53, 2019.

Lee, C. W.; Foo, Q. C.; Wong, L. V. \& Leung, Y. Y. An overview of maxillofacial trauma in Oral And Maxillofacial Tertiary Trauma Centre, Queen Elizabeth Hospital, Kota Kinabalu, Sabah. Craniomaxillofac. Trauma Reconstr., 10(1):16-21, 2017.

Maffia, F.; Fontanari, M.; Vellone, V.; Cascone, P. \& Mercuri, L. G. Impact of COVID-19 on maxillofacial surgery practice: a worldwide survey. Int. J. Oral Maxillofac. Surg., 49(6):827-35, 2020.

Marano, R.; Jadjisky, M.; de Mattos Filho, A. B.; Mayrink, G.; Araújo, S.; Oliveira, L.; Lemos, I. Z.; de-Souza, N. S.; Margato, R. G. \& Brandão, D. B. R. Epidemiological analysis of 736 patients who suffered facial trauma in Brazil. Int. J. Odontostomat., 14(2):25767, 2020.

Melián-Rivas, A.; Wallach-Weinstein, M.; Boin-Bakit, C. \& CarrascoSoto, R. Recommendations in the care of patients in maxillofacial surgery during the COVID-19 pandemic (SARS-CoV-2). Int. J. Odontostomat., 14(4):474-80, 2020. 
RAPOSO, A; NUÑEZ, C.; QUEZADA, G.; QUEZADA, L. \& CARRASO, A. Estudio descriptivo de las cirugías y hospitalizaciones en Cirugía Maxilofacial durante la pandemia en Chile. Experiencia del Hospital Barros Luco Trudeau. Int. J. Odontostomat., 15(1):71-76, 2021.

Pacheco, C.; Zapata, S. \& Núnez, C. Fracturas del tercio medio facial operadas en el Instituto Traumatológico de Santiago (Chile). 10 años de revisión. J. Oral Res., 1(2):71-6, 2012.

Pérez, G. H.; Donoso, H. T.; Mardones, M. M. \& Bravo, A. R. Epidemiology of maxillofacial surgical treatments in a public hospital in Chile: a 5-year retrospective study. Int. J. Odontostomat., 9(1):37-41, 2015.

Piombino, P.; Sani, L.; Committeri, U.; Carraturo, E.; Foderini, A.; Romano, A.; Orabona, GD.; Bonavolontà, P.; Abbate, V.; Califano, L. Guidelines and recommendations for patient management, surgical activities, and safety in the maxillofacial unit and head and neck department during COVID-19 pandemic. J. Craniofac. Surg., 2020. DOI: https://www.doi.org/10.1097/ SCS.0000000000006753

Raposo, A.; Preisler, G.; Salinas, F.; Muñoz, C. \& Monsalves, M. J. Epidemiología de las fracturas maxilofaciales tratadas quirúrgicamente en Valdivia, Chile: 5 años de revisión. Rev. Esp. Cir. Oral Maxilofac., 35(1):18-22, 2013.

World Health Organization (WHO). Coronavirus disease (COVID19). Data as received by WHO from national authorities, as of 11 October 2020, 10 am CEST 2020. Ginebra, World Health Organization, 2020. Disponible en: https://www.who.int/docs/ default-source/coronaviruse/situation-reports/20201012-weeklyepi-update-9.pdf

van der Tas, J.; Dodson, T.; Buchbinder, D.; Fusetti, S.; Grant, M.; Leung, YY. 2020. The global impact of COVID-19 on craniomaxillofacial surgeons. Craniomaxillofac. Trauma Reconstr., 2020. DOI: https://www.doi.org/10.1177/ 1943387520929809

Yang, Y.; Soh, H. Y.; Cai, Z. G.; Peng, X.; Zhang, Y. \& Guo, C. B. Experience of diagnosing and managing patients in oral maxillofacial surgery during the prevention and control period of the new coronavirus pneumonia. Chin. J. Dent. Res., 23(1):5762, 2020.

Yuvaraj, V. Maxillofacial infections of odontogenic origin: epidemiological, microbiological and therapeutic factors in an Indian population. Indian J. Otolaryngol. Head Neck Surg., 68(4):396-9, 2016.

Zimmermann, M. \& Nkenke, E. Approaches to the management of patients in oral and maxillofacialsurgery during COVID-19 pandemic. J. Craniomaxillofac. Surg., 48:521-6, 2020.
Dirección para correspondencia:

Araceli Raposo Castillo

Unidad de Cirugía y Traumatología Maxilofacial

Servicio de Cirugía

Hospital Barros Luco Trudeau

Santiago

CHILE

E-mail: araceliraposo@gmail.com 